evidence to recommendation framework in GRADE and clarification of issues that relate to laboratory validity parameters.

\section{NONRANDOMISED STUDIES AS A SOURCE OF COMPLEMENTARY, SEQUENTIAL OR REPLACEMENT EVIDENCE FOR RANDOMISED CONTROLLED TRIALS IN SYSTEMATIC REVIEWS AND GUIDELINES}

${ }^{1} \mathrm{H}$ Schünemann, ${ }^{2} \mathrm{P}$ Tugwell, ${ }^{3} \mathrm{~B}$ Reeves, ${ }^{1,4} \mathrm{E}$ Akl, ${ }^{1} \mathrm{~N}$ Santesso, ${ }^{1} \mathrm{~F}$ Spencer, ${ }^{5} \mathrm{~B}$ Shea, ${ }^{2} \mathrm{G}$ Wells, ${ }^{6} \mathrm{M}$ Helfand. ${ }^{1} \mathrm{McM}$ aster University, Hamilton, Canada; ${ }^{2}$ University of Ottawa, Ottawa, Canada; ${ }^{3}$ University of Bristol, Bristol, UK; ${ }^{4}$ American University of Beirut, Beirut, Lebanon; ${ }^{5}$ Vu University Medical Center, Amsterdam, Netherlands; ${ }^{6}$ Oregon Health \& Science University, Oregon, USA

\section{0:1136/bmjqs-2013-002293.90}

Background The terms applicability, generalizability, external validity, transferability generally describe one overarching theme: can available research evidence be utilised to answer the health care questions at hand, ideally supported by a judgement about the degree of confidence in this utilisation. This concept has been called directness.

Objectives To offer conceptual and practical guidance to those judging directness and using research evidence from non-randomised studies (NRS).

Methods We used a literature review and feedback from participants of a workshop funded by the Agency for Healthcare Quality and Research and the Cochrane Collaboration.

Results Guideline developers can use NRS as a source of complementary, sequential or replacement evidence for randomised controlled trials (RCTs) by focusing on judgements about the population, intervention, comparison and outcomes. They use NRS to complement judgements about inconsistency, the rationale and credibility of subgroup analysis, baseline risk estimates for the determination of absolute benefits and downsides, and the directness of surrogate outcomes. Authors use NRS as sequential evidence to provide evidence when the evidence from RCTs is insufficient (e.g. long-term harms). Use of evidence from NRS may also replace RCT evidence when RCTs provide indirect evidence but NRS provide overall higher quality, direct evidence. We developed a simple tool and algorithm to make judgements about indirectness more transparent.

Discussions These judgements need to be made in the context of other quality of evidence domains.

Implications for Guideline Developers/Users The transparency of the framework will support interaction with those making health care decision and policy.

\section{APPRAISING IMPLEMENTABILITY DURING THE DEVELOPMENT PROCESS RESULTED IN GUIDELINE REVISION}

${ }^{1,2} \mathrm{~S}$ van der Veer, ${ }^{1} \mathrm{~A}$ Simon, ${ }^{3} \mathrm{D}$ Weismann, ${ }^{4} \mathrm{G}$ Lehner, ${ }^{5} \mathrm{~L}$ Coentrão, ${ }^{1} \mathrm{D}$ Dongelmans, ${ }^{6} \mathrm{~S}$ van Laecke, ${ }^{6} \mathrm{~B}$ Lapauw, ${ }^{7} \mathrm{~A}$ Ortiz, ${ }^{8} \mathrm{~A}$ Schiller, ${ }^{9} \mathrm{~V}$ Tesar, ${ }^{2,6}{ }^{6} \mathrm{E}$ Nagler. ${ }^{1}$ Academic Medical Center, Amsterdam, The Netherlands; '2European Renal Best Practice, Ghent, Belgium; ${ }^{3}$ University Hospital Würzburg, Würzburg, Germany; ${ }^{4}$ Medical University Innsbruck, Innsbruck, Austria; ${ }^{5}$ University of Porto Sao Joao Hospital Centre, Porto, Portugal; ${ }^{6}$ University Hospital Ghent, Ghent, Belgium; ${ }^{7}$ IIS-Fundacion Jimenez Diaz, Madrid, Spain; ${ }^{8}$ Emergency Hospital Timisoara, Timisoara Romania General University Hospital, Prague, Czech Republic

\section{0:1136/bmjgs-2013-002293.91}

Background The GuideLine Implementability Appraisal (GLIA) instrument has been suggested for identifying potentially remediable implementability issues during the guideline development process.
Objective To explore to what extent using GLIA during the development process would result in guideline revision before publication.

Methods The development process of the European hyponatremia guideline -coordinated by European Renal Best Practice - was our study context. Using the GLIA web-tool, eleven clinicians and methodologists from eight countries individually appraised 27 guideline statements. In a face-to-face consensus meeting, four GLIA panelists and one guideline development group (GDG) representative summarised potential implementability issues. The GDG discussed these issues, and revised the guideline if deemed necessary.

Results We identified 33 issues; the GDG accepted 26 as potentially hampering implementability. This resulted in statement reformulation with $(n=5)$ and without $(n=10)$ influencing clinical content, adding or (re)moving entire statements $(n=8)$, and adding information to tables or rationales $(n=3)$. The majority of issues declined by the GDG $(n=7)$ addressed clinical situations that were covered elsewhere in the guideline or were considered to be uncommon.

Discussion Using GLIA during the development process resulted in a revised guideline. We felt that GDG representation in the consensus meeting optimize our appraisal process.

Implications for Guideline Developers/Users Guideline organizations may want to consider incorporating GLIA into their development process. This may raise GDGs' awareness of potential implementability issues, and allow revision of the guideline accordingly prior to publication. Future research should explore the effect of GLIA-based revisions on implementability as assessed by guideline users.

\section{GUIDELINE IMPLEMENTABILITY APPRAISAL (GLIA) IN US NATIONAL GUIDELINES}

${ }^{1} \mathrm{~W}$ Chan, ${ }^{2} \mathrm{~T}$ Pearson. ${ }^{1}$ Kaiser Permanente, Northwest, Portland, USA; ${ }^{2}$ University of Rochester, Rochester, NY, USA

\section{0:1136/bmjqs-2013-002293.92}

Background Guidelines must be implemented in order to impact health outcomes. Identifying and addressing potential barriers to implementation during guideline development can improve implementability.

Objectives To describe the processes and results of embedding guideline implementability appraisal, into prominent US cardiovascular disease risk reduction guidelines.

Methods The GuideLine Implementability Appraisal (GLIA) tool (Yale Center for Medical Informatics), was integrated into the guideline development processes of a US national-level organisation. A member of the Implementation Science Work Group (ISWG) with prior experience in GLIA appraisals trained the Guideline Development Teams (GDTs), early in the guideline development process, with the intent of raising awareness of potential barriers to implementation so they might be addressed during guideline development. Formal GLIA appraisals were performed on the drafts of the guideline reports, by members of the ISWG, as well as volunteers from outside the guideline programme. To minimise interference with timelines, appraisals were carried out and written reports returned to the GDTs within 2 weeks of release of the draft reports.

Results A number of potential barriers to implementation were identified in the draft reports, such as: use of inexplicit terms in recommendation language, inconsistency of thresholds and terms used within a guideline, unclear applicability of assessment 
or treatment recommendations in specific subgroups. GDTs considered GLIA appraisal findings when they revised their reports and found the GLIA appraisals helpful in creating more implementable guidelines.

Implications for Guideline Developers/Users GLIA training for GDTs, and formal use of the GLIA tool help produce more implementable guidelines.

\section{PARTIALLY UPDATING A GUIDELINE TO IMPROVE ITS IMPLEMENTATION}

S Warttig, N Elliott. National Institute for Health and Clinical Excellence, Manchester, UK

\section{0:1136/bmjgs-2013-002293.93}

Background We were commissioned to partially update a clinical guideline. The remit was to develop new service delivery recommendations to support implementation of the guideline whilst leaving the existing clinical recommendations unchanged.

Objectives To describe the approach taken in partially updating a guideline to improve its implementation. To discuss the problems encountered and possible solutions.

Methods At the time, there was limited guidance on conducting service delivery evidence reviews. A methodology was developed and agreed by the developers, the NICE Methodology Team and the GDG which aimed to ensure the process was as robust, reproducible and transparent as possible.

Results Limited evidence was identified using the agreed methodology. This prevented identification of successful service delivery models. It also became apparent that that some of the implementation issues were embedded in the original guideline recommendations, and these could not be changed.

Discussion The methodology used could not adequately address the implementation issues, as it was not possible to amend any of the problematic recommendations, or describe a method of service delivery that was clinically and cost effective. Agreement could not be reached on how to progress with developing the recommendations, and so a decision was made to cease publication of the service delivery recommendations.

Implications for Guideline Developers/Users Partial updates are more challenging for guidelines requiring implementation support and should: 1) Go through a process to assess the issues before deciding how guideline should be updated. Or 2) Come with a remit to enable the developers to amend the recommendations for which implementation support is sought.

\section{ENHANCING THE UPTAKE OF CLINICAL PRACTICE GUIDELINES: THE DEVELOPMENT OF A GUIDELINE IMPLEMENTABILITY TOOL (GUIDE-IT)}

M Kastner, J Versloot, L Hayden, A Chatterjee, O Bhattacharyya. Li Ka Shing Knowledge Institute of St. Michael's Hospital, Toronto, Canada

\section{0:1136/bmjqs-2013-002293.94}

Background Guidelines have the potential to facilitate implementation of evidence into practice but this has not been consistently achieved. We developed a guideline implementability tool (GUIDE-IT), which can assess the implementability of guideline recommendations.

Objective To determine if GUIDE-IT can improve the Language and Format of guideline recommendations.
Methods Using a mixed-methods approach to develop GUIDEIT, we conducted 1) a Realist Review of guideline factors influencing uptake, and used its results to build a conceptual model of guideline implementability; 2) qualitative interviews with 20 family physicians to determine factors influencing guideline uptake and to obtain input on tool design; 3) created a prototype and conducted validity assessments with experts in guideline development and human factors. GUIDE-IT was then pilot tested with the Canadian Diabetes/Paediatric Associations (CDA, CPS) to determine its potential for assessing the implementability of guideline recommendations.

Results Pilot testing with CDA and CPS developers showed that factors across 4 sub-domains of Language (clarity, simplicity, specificity, and actionability) and 3 sub-domains of Format (presentation, components, and multiple versions) were applicable for modifying recommendations. GUIDE-IT was feasible to use by guideline developers to identify implementability problems and to improve recommendations.

Discussion GUIDE-IT is based on a robust evidentiary base with the potential to improve guidelines. Next steps include evaluating GUIDE-IT in a controlled trial to determine its impact on end-user clinical decision making.

Implications for Guideline Developers/Users GUIDE-IT has potential to be a practical tool for developers to improve the language and format of guideline recommendations.

\section{IDENTIFYING, DESCRIBING AND EVALUATING GUIDELINE IMPLEMENTABILITY TOOLS}

${ }^{1} \mathrm{~J}$ Cheng, ${ }^{1} \mathrm{~A}$ Gagliardi, ${ }^{2} \mathrm{M}$ Brouwers, ${ }^{3} \mathrm{O}$ Bhattacharyya. ${ }^{1}$ University Health Ne; ${ }^{2}$ Department of Oncology, McMaster University, Hamilton, Canada; ${ }^{3}$ St. Michael's Hospital, Toronto, Canada

\section{0:1136/bmjqs-2013-002293.95}

Background Research shows that guidelines are more easily translated to practice when accompanied by information that helps users accommodate, implement and evaluate use of the recommendations. Guidelines vary in whether and how they offer such information, which we refer to as guideline implementability tools (GItools).

Objectives To identify, describe and evaluate exemplar GItools that address Resource Implications, Implementation and Evaluation, and suggest how they could be improved.

Methods GItools were identified in several sources (guidelines, Medline, professional organisation web sites, Implementation Science, Internet, expert referrals) and two individuals independently assessed each on criteria recommended by G-I-N members: purpose statement, instructions for use, citations for source of content, and how it was developed.

Results The search produced 228 potential tools. Of these 94 were ineligible and 63 met no assessment criteria. Of the remaining 71 tools, 13 (18.3\%), 24 (33.8\%), 23 (32.4\%) and 11 (15.5\%) met 1, 2, 3 and 4 criteria, respectively; and 57 (80.3\%), $37(52.1 \%), 41(57.7 \%)$ and $41(57.7 \%)$ provided purpose, instructions, citations and development details, respectively. Most tools addressed Implementation (44, 62.0\%). Twenty-eight (39.4\%) were guideline-specific and $43(60.6 \%)$ were generic.

Discussion Few GItools met all assessment criteria. GItools could be more informative across all criteria. Few GItools were available to help users assess resource needs or evaluate guideline use. Many GItools were applicable to a variety of guidelines.

Implications We identified a number of ways to improve GItools. Collaborative development and sharing of both generic 